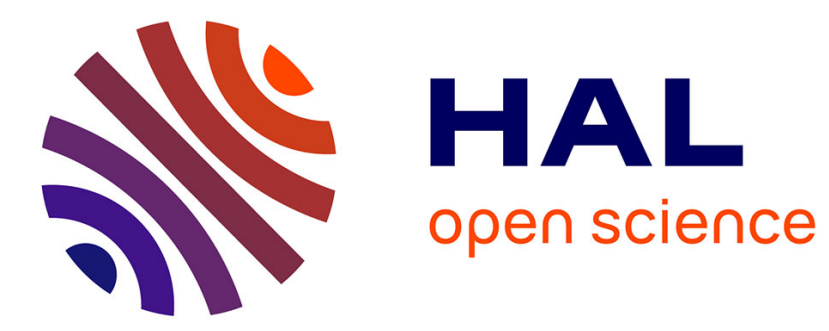

\title{
Scanning transmission electron microscopy
}

L. Brown

\section{To cite this version:}

L. Brown. Scanning transmission electron microscopy. Journal de Physique IV Proceedings, 1993, 03

(C7), pp.C7-2073-C7-2080. 10.1051/jp4:19937331 • jpa-00251978

\section{HAL Id: jpa-00251978 https://hal.science/jpa-00251978}

Submitted on 1 Jan 1993

HAL is a multi-disciplinary open access archive for the deposit and dissemination of scientific research documents, whether they are published or not. The documents may come from teaching and research institutions in France or abroad, or from public or private research centers.
L'archive ouverte pluridisciplinaire HAL, est destinée au dépôt et à la diffusion de documents scientifiques de niveau recherche, publiés ou non, émanant des établissements d'enseignement et de recherche français ou étrangers, des laboratoires publics ou privés. 


\title{
Scanning transmission electron microscopy
}

\author{
L.M. BROWN \\ Cavendish Laboratory, Madingley Road, Cambridge CB3 OHE, U.K.
}

Since its practical realisation by Crewe, STEM utlising a field emission source has become a versatile technique offering many types of signal which can reveal chemical and structural information at the atomic or near-atomic level. Chemical information can be obtained from the electron energy loss spectrum at a spatial resolution which now permits analys is of individual atomic columns. Furthermore the fine structure in these spectra can in many cases rival the structure in $\mathrm{X}-\mathrm{ray}$ absorption spectra from synchrotron sources. The highly coherent source permits phase relations to be deduced from convergent beam patterns and promises to allow images in both amplitude and phase to be formed at a level of resolution not subject to the usual diffraction and aberration limit. There are a very wide range of applications within solid state science. Finally, the use of the beam to write tailor-made structures for electron optical components and other purposes introduces a wide range of activity requiring FEG STEM.

With the advent of 'atomic column microanalysis', achieved by Pennycook and his team at Qak Ridge National Laboratory (1), the STEM revolution initiated by Crewe (2) may be said to be complete. Atomic column microanalysis utilises a modification of Crewe's idea of $Z$-contrast to image atomic columns one by one, and at each column a high-quality electron energy loss spectrum is acquired so that the chemical composition of the column can be assessed. The modified Zcontrast technique is based on a high angle annular dark field detector, the electrons having been deflected through a sufficiently large angle that the atoms scatter independently and incoherently. In crystalline materials a large scattering angle is required, several degrees, as first pointed out by Howie and employed to enhance the images of small catalyst particles (3). The electron energy loss spectroscopy is facilitated by the use of a CCD detector which allows low noise parallel detection with high efficiency. These detector systems are designed and built by McMullan and coworkers (4). 
The application of these techniques depends crucially on a clear understanding of the effective spatial resolution rEELS associated with the electron energy loss spectrum. If we add independent contributions in quadrature, following earlier work (5), we find

$$
\mathrm{rEELS}^{2}=r_{0}^{2}+(\alpha \mathrm{t})^{2}+\mathrm{b}^{2}
$$

where $r_{0}$ is a measure of the unbroadened probe size, $\alpha$ is the broadening associated with a point probe propagating into a collector aperture of semiangle $\alpha$ and $b$ is the impact parameter associated with the energy loss event which may be conveniently estimated from graphs presented by Pennycook (6). In effect, for $100 \mathrm{kV}$ electrons, and typical apertures of $10 \mathrm{mr}$, losses of $200 \mathrm{~V}$ or more will reduce $b$ to less than $0.2 \mathrm{~nm}$. The most important point concerns the second term. If the aperture size is $10 \mathrm{mr}$, it contributes $0.2 \mathrm{~nm}$ if the foil is $20 \mathrm{~nm}$ thick or more, implying that the foils must be thinner than this to achieve column-by-column analysis. However. Pennycook and Jesson (7) argue that especially for zone-axis orientations of the thin crystalline foil, channeling of the electrons in the probe prevents the probe from spreading, reduces the value of $\alpha$ one expects from the electron optics, and enables good $Z$-contrast to be achieved in thicker foils. It seems therefore that under favourable circumstances the term dependent upon the foil thickness may be suppressed and column-bycolumn analys is may be applied to thicker specimens. Surely at this point in the development of these ideas one should be cautious; for example, in amorphous materials and at grain boundaries, where no channeling is possible, probe spreading must occur. The probe spreading for unapertured spectroscopy, such as EDX, occurs because of medium to large angle incoherent elastic scattering, which because it is outside microscope apertures is largely responsible for absorption in conventional transmission electron microscopy. The channeling idea of Pennycook and Jesson relies upon the attractive potential presented by the atomic columns to the fast electrons, sharpening the probe when it is located over an atomic column. These are the orientations in the T.E.M. for which anomalous absorption occurs because the fast electron wave functions are preferentially scattered near the atomic cores. Thus one can understand how the channeling idea works because such orientations enhance Rutherford scattering near the atomic nulei. Precisely such orientations also enhance the higher energy inelastic transitions which depend upon core excitations of the atoms. However, it is important to avoid degradation of the spectrum by multiple inelastic (plasmon) scattering. Plasmon excitation will degrade both the energy resolution and the spatial resolution, by amounts which are significant in terms of this discussion. For this reason alone it is inadvisable to use foils thicker than a few tens of nanometres. What one can say is that the combination of instrumentation available at the Oak Ridge group will quickly allow these questions to be settled; furthermore, even if the technique is limited to thinner specimens than one might hope, it is still likely to be the most powerful high resolution analytical technique available for some time. In the future it is just conceivable that in robust specimens of heavy atoms enough signal will be available to image single atoms, so that a stereo pair will reveal by $Z$-contrast the three dimensional structure, perhaps even irregular grain boundary structure. 


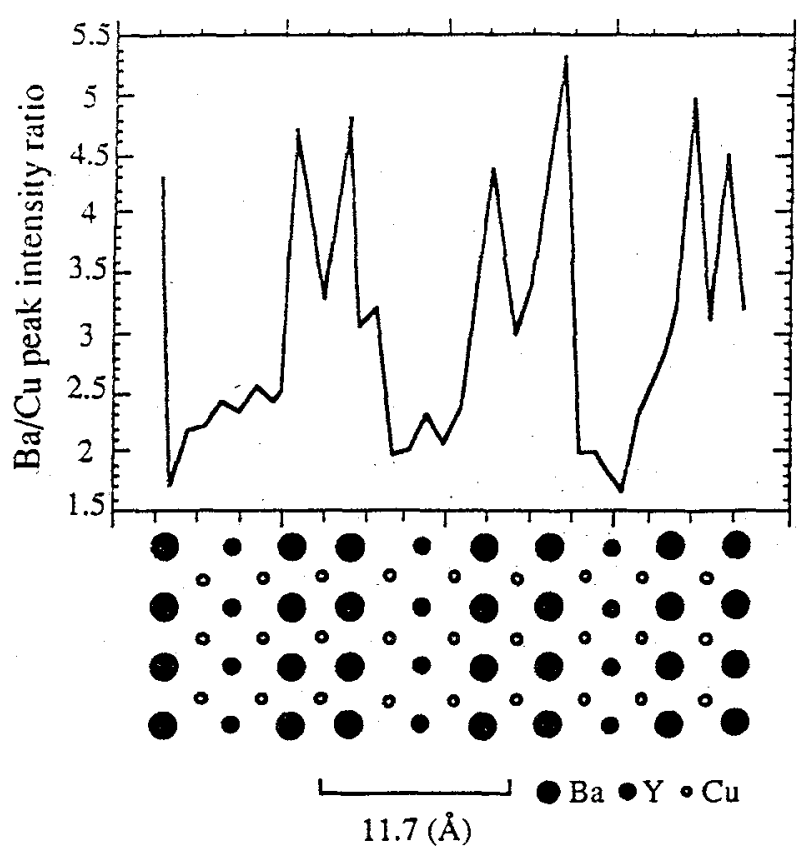

Fig. 1. An example of atomic column microanalysis, reproduced by kind permission of N. D. Browning and S. J. Pennycook (1).

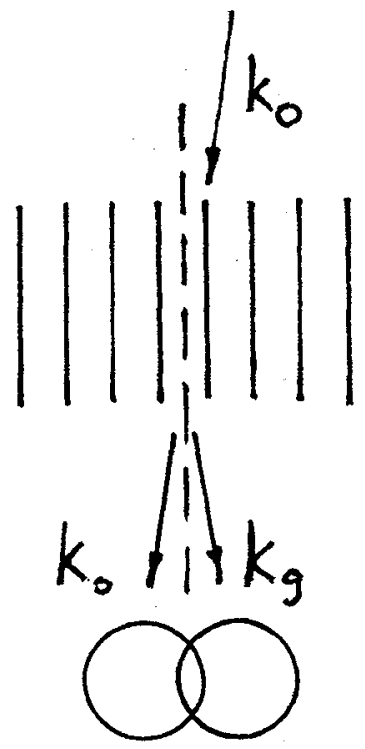

Fig. 2. A plane incident wave, vector $\mathrm{k}_{0}$, inclined to the optic axis (dashed line) produces a diffacted beam $\mathrm{kg}$ by interaction with "Bragg planes" or any periodicity in the projected specimen lattice potential. A wide enough convergent beam will produce overlapping discs, whose relative phase can be determined from the overlap region. 
An example drawn from recent work by Browning and Pennycook (1) is shown in Fig. 1. The oxide superconductor $\mathrm{YBa}_{2} \mathrm{Cu}_{3} \mathrm{O}_{7}$ is viewed parallel to its basal planes and the $\mathrm{Ba} / \mathrm{Cu}$ ratio is measured from the energy loss spectrum. The column-by-column variation is clearly demonstrated, so that analysis is being achieved on a $0.2 \mathrm{~nm}$ scale; quantitative analysis must await further experiments to estimate the beam broadening.

These techniques are effective because they rely upon incoherent imaging. All the interference fringes between the various Bragg reflections are suppressed by making the inner angle of the detector large enough that the Bragg reflections are washed out by thermal vibrations. It is remarkable that FEG STEM permits also the opposite point of view: because the probe is bright, coherent microdiffraction can be obtained from nanometre sized areas of the foil, and interference between the resulting discs in the convergent beam pattern can be seen. Thus the 'phase problem' is solved and the structure of the sampled volume can in principle be obtained directly by inverse Fourier transform for each probe position. In addition, scanning the probe will permit complete knowledge to be obtained about the fast electron wave function at the exit surface of the specimen. This is available at a level of detail which is not subject to the Rayleigh resolution limit, achieving so-called 'super-resolution'.

The aim is briefly then to improve the resolution by utilising data in the microdiffraction plane, because such data is not limited by the transfer characteristics of the lens. It contains information on the spatial frequencies (and, if coherent diffraction can be assumed, on their relative phases) which correspond to spacings much less than the conventional resolution limit of the microscope. To quote from a recent paper by Rodenburg and his team (8): "if a sufficiently redundant data set is processed consisting of either (i) all coherent microdiffraction patterns available in the STEM collected as a function of many equally-spaced probe positions, or (ii) all coherent bright-and dark-field images available in the CTEM collected as a function of many equally-spaced illumination angle conditions, then it is possible to remove all the degrading transfer characteristics of the objective lens and solve for the exit wavefunction from the specimen in complex amplitude at much improved resolution". This remarkable theorem seems to include the Gerchberg-Saxton algorithm (9) which by consideration of both the image and diffraction plane enables in principle the determination of the phase of the wave function.

A simple example may help to illustrate Rodenburg's ideas. Consider Fig. 2, which shows a plane wave incident upon a weak phase object. The incident wave is tilted with respect to the optic axis so that it produces a diffracted wave equally but oppositely inclined to the optic axis of the microscope. In such conditions, because phase changes due to spherical aberration and defocus are even functions of angle, the relative phase of the incident and diffracted beams is independent of the lens (to within standard approximations for lens aberrations) and depends only on the position of the specimen. Now if we make use of reciprocity, and imagine a convergent beam incident from below with an angular width large enough to encompass both the incident and diffracted directions, then where the resulting discs overlap their relative phase can be 

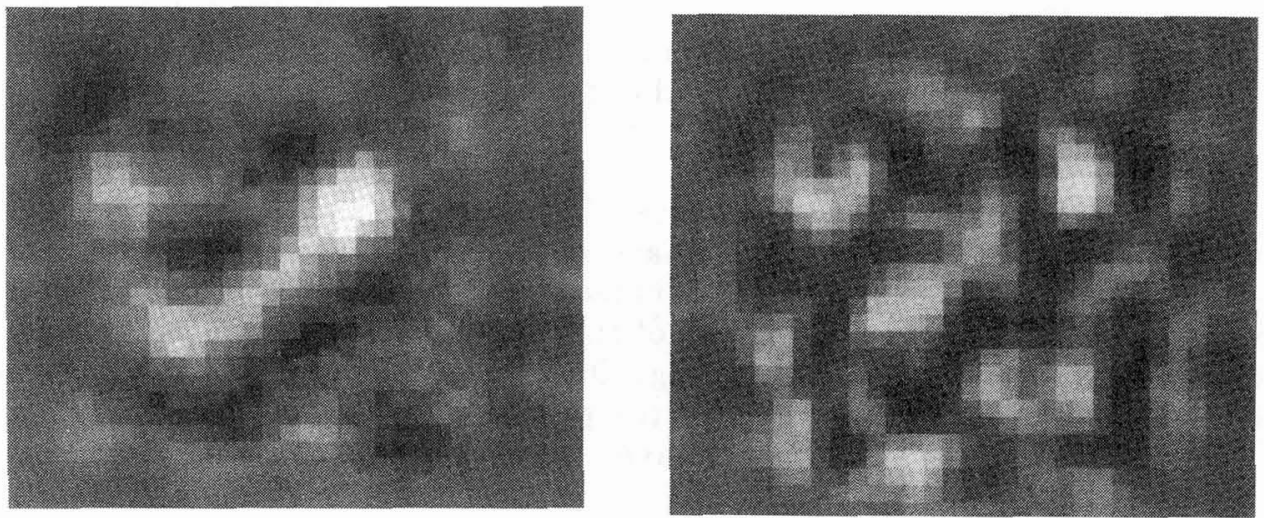

Fig. 3. An example of resolution doubling by kind permission of Rodenburg, McCallum, and Nellist (8). The left hand figure shows the bright-field image: the sampling pixels are $0.19 \mathrm{~nm}$ square and the objective aperture $4 \mathrm{mrad}$, giving a nominal resolution of $0.93 \mathrm{~nm}$. After processing the data set, the phase image shows the expected double resolution. Repeated independent experiments confirm that the result is reproducible.
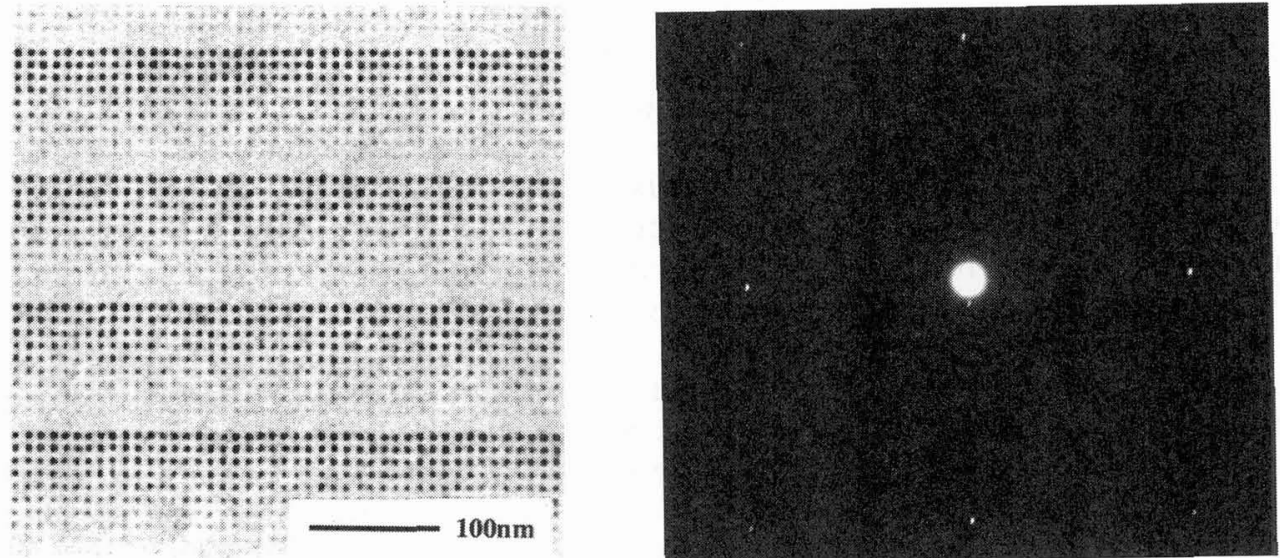

Fig. 4. A digital image of part of a $100 \times 100$ wedge grating formed by electron beam writing using the VG HB501 STEM on $\mathrm{AlF}_{3}$, together with its diffraction pattern as recorded at $200 \mathrm{kV}$. The asymmetry in the pattern demonstrates that it is acting as a phase grating. (After ref. (10)). 
determined. Once two such discs have been phased, an entire array of overlapping discs can be phased and in principle the amplitude and phase of the entire exit wavefunction - viewed as a Fourier sum of its diffracted components - can be built up.

Can these ideas be made to work in practice? The first successful attempt to present both amplitude images and phase images at double the recorded bright field resolution has recently been published (8); see Fig. 3. But as Rodenberg et. al. point out (8), there is still a factor of three to go before his super resolution method' overtakes conventional imaging. Only technical obstacles seem to stand in the way of overcoming this. Even if the path should prove more difficult than seems likely at the moment, the phase component obtainable from the new methods promises to solve structures directly, particularly perhaps amorphous structures, and high resolution magnetic structures which have so far proved intractable. There seems little doubt that these methods will ultimately overtake conventional high resolution techniques, which are increasingly costly and cumbersome.

The use of optical filtering to enhance images is a standard technique. Rodenburg's method essentially processes all the information both in the diffraction plane and in the image plane to produce an optimised image both in amplitude and phase. However it is now possible to devise phase plates which could in principle be used in the column of the microscope itself to enhance phase contrast; for example, to produce in-focus images of weak phase objects. This is made feasible by the use of electron beam writing to fabricate phase gratings of nanometre size directly in the STEM (10). A thin film of an inorganic resist is used, typically $\mathrm{AlF}_{3}$. The dwell time of the focussed probe is controlled, so that holes of different depths are drilled. The film is thin enough that it produces very little amplitude contrast, to a good approximation only phase contrast. A demonstration of this is shown in figure (4): a phase grating incorporating a phase gradient produces the assymetric diffraction pattern shown inset. The basic spacing in the pattern corresponds to the grating spacing. However near the origin (and near every spot in the pattern) there is a sideband on one side only which corresponds to beam bending towards the thicker holes. This method can be used to make biprisms producing a deflection similar in magnitude to the electrostatic biprisms now used for electron holograpliy. Such prisms are located in an image plane to produce overlapping images at subsequent image planes in the column. It is in principle also possible to fabricate a phase plate to enhance predetermined structures, for example molecules of a given shape, directly by intervention in the diffraction plane of the microscope. The phase plates are written in the STEM and transferred to the conventional microscope where at the much lower current densities they are sufficiently stable to be used as electron optical components.

The STEM now is a powerful and flexible tool for solid state science, with applications to many areas, both pure and applied. A recent survey of work going on in the Cavendish Laboratory has been published (11). However one important new application to the characterisation of colloidal particles has been opened up by J. Yuan (private communication). Colloidal systems such as paints 
rely upon the control of interparticle forces, which comprise attractive Van der Waals forces in addition to other forces caused by charged double layers, polymeric surface coatings, etc. Because Van der Waals forces result from correlated dipole moments, they depend directly upon the dielectric functions measured by EELS. According to Yuan, the Lifshitz theory for the strength of the $V$ an der Waals force exerted by a particle $A$ embedded in medium $B$ is dependent upon a quantity

$$
\Delta_{\mathrm{BA}}(\mathrm{i} \omega)=\frac{2}{\pi} \int \frac{\operatorname{Im}\left(\Delta_{\mathrm{BA}}\left(\omega^{\prime}\right)\right) \omega^{\prime} \mathrm{d} \omega^{\prime}}{\omega^{2}+\omega^{\prime 2}}
$$

where $\operatorname{Im}\left(\Delta_{\mathrm{BA}}(\omega)\right)$ is precisely the function measured by electron energy loss spectroscopy at the interface between medium $A$ and medium $B$

$$
\Delta(\omega)=\frac{\varepsilon_{A}(\omega)-\varepsilon_{B}(\omega)}{\varepsilon_{A}(\omega)+\varepsilon_{B}(\omega)}
$$

Thus there is a direct link between the electron energy loss spectrum and interparticle forces. Contributions to the Van der Waals forces from the infrared losses cannot be assessed by EELS because of inadequate energy resolution, but the ultraviolet part of the spectrum makes a more important contribution, particularly close to the interface, and this is where STEM has a vital role to play. It is possible to observe quite complex particles, with various surface coatings, and from the energy loss spectroscopy to estimate the strength of the Van der Waals term in the expression for the forces between the particles. Preliminary work on $\mathrm{TiO}_{2}$ particles seems to indicate that in a practical case this program can be carried out, the expected forces agreeing with those measured directly.

\section{REFERENCES}

(1) N. D. Browning, M. F. Chisholm, and S. J. Pennycook, to be published in 1993 Analytical Electronmicroscopy Workshop, Los Angeles; see also N. D. Browning and S. J. Pennycook 1993 Proc. Fall Meeting of the Mat. Res. Soc., Boston, Mass., Nov-Dec 1992. Many other papers are in preparation for Microbeam Analysis and Materials Research Society.

(2) A. V. Crewe, 1971, in 'Electron Microscopy in Material Science', ed. U. Valdre, Academic Press, London.

(3) M. M. J. Treacy, A. Howie and S. J. Pennycook, 1980 Inst. Phys. Conf. Ser. No. 52, p.261, Ed. T. Mulvey, Inst. of Physics, Bristol and London. 
(4) S. D. Berger and D. McMullan, 1989 U1tramicroscopy 28, pp 122-125; see also D. McMullan, J. M. Rodenburg, Y. Murooka, and A. J. McGibbon, Inst. Phys. Conf. Ser. 98 (1990) pp 55-58, Ed. P. J. Goodhew and H. Y. Elder, Inst. Phys. Bristol and New York.

(5) S. A. Collett, L. M. Brown, and M. H. Jacobs, 1984 Inst. Phys. Conf. Ser. 68, pp 103-106, Ed. P. Doig, Inst. Phys. Bristol and London.

(6) S. J. Pennycook, 1982, Contemp. Phys. 23, pp 371-400.

(7) S. J. Pennycook and D. E. Jesson, 1991 Ultramicroscopy 37, pp 14-38.

(8) J. M. Rodenburg, B. C. McCallum and P. D. Nellist, 1003 Ultramicroscopy 48, pp304-314.

(9) R. W. Gerchberg and W. O. Saxton 1972, Optik, 35 pp237-246.

(10) Y. Ito, A. L. Bleloch, J. H. Paterson, and L. M. Brown, 1993, presented at the John M. Cowley Symposium, Tempe, Arizona; to be published in Ultramicroscopy.

(11) A. J. McGibbon et. al., 1993 Microscopy Research and Technique, 24, pp299-315. 